\title{
Simple Algorithms for Estimating the Symbol Timing Offset in DCT-Based Multicarrier Systems
}

\author{
Fernando Cruz-Roldán $\left(\mathbb{D},{ }^{1}\right.$ José Piñeiro-Ave $\mathbb{D}$, ${ }^{1}$ José L. Rojo-Álvarez $\mathbb{D},{ }^{2}$ \\ and Manuel Blanco-Velasco (i) ${ }^{1}$ \\ ${ }^{1}$ Department of Signal Theory and Communications (EPS), Universidad de Alcalá, 28805 Alcalá de Henares, Madrid, Spain \\ ${ }^{2}$ Department of Signal Theory and Communications, Universidad Rey Juan Carlos, 28943 Fuenlabrada, Madrid, Spain \\ Correspondence should be addressed to Fernando Cruz-Roldán; fernando.cruz@uah.es
}

Received 4 April 2018; Accepted 28 June 2018; Published 15 July 2018

Academic Editor: Takayuki Ito

Copyright (C) 2018 Fernando Cruz-Roldán et al. This is an open access article distributed under the Creative Commons Attribution License, which permits unrestricted use, distribution, and reproduction in any medium, provided the original work is properly cited.

\begin{abstract}
Multicarrier modulation based on discrete cosine transform (DCT-MCM) is a candidate technique for future wireless communication. DCT-MCM provides a better usage of the spectrum, allowing for much lower out-of-band radiation than conventional OFDM. In this paper, we address the problem of correcting the symbol timing offset in DCT-MCM. Two sliding window-based correlation algorithms are proposed. The new approaches consider a prefix and a suffix that are inserted into each data symbol, which are the replica of part of the useful data. The performance of the proposed approaches is verified and tested, and their usefulness and effectiveness are illustrated by computer simulations.
\end{abstract}

\section{Introduction}

The next generation of communication systems (5G and beyond) will employ higher bandwidths and data rates that are able to provide enhanced data services to users, also improving the efficiency of spectrum usage. Consequently, channel partitioning methods will be the dominant medium access techniques due to their appealing features [1], such as higher system performance and spectrum efficiency, typically measured in (bit/s)/Hz per unit area or cell.

Discrete multitone (DMT) modulation and orthogonal frequency division multiplexing (OFDM) are examples of channel partitioning techniques that employ the strategy of replicating the data into each transmitted symbol. This replica of part of the data, usually referred to as redundant samples, leads to a channel partitioning that provides a set of ideally independent subchannels in which channel equalization can be easily performed [1]. DMT and OFDM are efficiently implemented using discrete Fourier transforms (DFTs). These techniques, however, have drawbacks, such as a sensitivity to offsets in time and carrier frequency. Moreover, the temporal pulse shape of DFT is a rectangular window, thus DFT-based multicarrier modulation (DFTMCM) suffers from high side-lobe radiation.

As alternatives to DFT-based solutions, several authors have proposed the use of discrete cosine transforms (DCTs) [2-6]. DCT-based multicarrier modulation (DCT-MCM) offers benefits such as excellent spectral compaction and energy concentration and less intercarrier interference leakage to adjacent subcarriers or that DCT uses only real arithmetic [2,3]. Furthermore, DCT-MCM provides a better usage of the spectrum allowing for much lower out-ofband radiation. As has been widely reported (e.g., [3]), the bandwidth of a DCT-MCM system can be only half of the bandwidth required by DFT-MCM with the same number of subcarriers.

In any multicarrier modulation (MCM) systems, symbol timing estimators play an important role in the receiver to find the start of the symbol of the received signal. Numerous methods for DFT-MCM have been proposed since the publication of the maximum likelihood (ML) estimation algorithm in an additive white Gaussian noise (AWGN) channel and the method of Schmidl and Cox [7-9]. We refer the reader to the studies in [10-12] for a 
detailed list of recent synchronization approaches and their implementations in some hardware/software environments for different real-world applications. Unfortunately, this field has received less attention for the DCT-MCM, even though synchronization is also crucial and critical. In [13], two joint maximum likelihood frequency offset and phase offset estimators, considering only AWGN channels, were presented. More recently, a new symbol synchronization method for optical fast OFDM is reported in [14]. As a result, the problem of a feasible technique for DCT-MCM still remains open.

The main novelty of this paper is the proposal of simple algorithms to carry out precise symbol timing estimation for DCT-MCM. The new approaches exploit the redundancy inserted as both left and right extensions in DCTbased systems. It is shown by computer simulations that the proposed approaches yield efficient and feasible time estimation solutions, providing satisfactory results in several communication scenarios.

The rest of this paper is organized as follows. In Section 2, the system model for the considered multicarrier transceivers is described. Section 3 presents the proposed algorithms for DCT2e and DCT4e-based systems. Section 4 provides a performance evaluation of the proposed algorithms and its comparison to DFT-based systems, and, finally, concluding remarks are given in Section 5.

\section{System Model}

Figure 1 is a general block diagram to implement MCM. At the transmitter, the data are processed by an $N$ point inverse transform $\mathbf{T}_{a}^{-1}$, with $N$ being the number of subchannels or subcarriers. At the receiver, a discrete transform $\mathbf{T}_{c}$ is performed.

In DFT-MCM (e.g., OFDM/DMT systems), $\mathbf{T}_{a}^{-1}$ is an IDFT, the redundancy of length $N_{G}$, e.g., a cyclic prefix (CP) (see Figure 2(a)), is introduced at the beginning of each $N$ length data symbol $x[n]$ to be transmitted, $h_{p f}[n]$ is a timedomain equalizer that shortens the effective channel $h_{c h}[n]$ to an appropriate length, $\mathbf{T}_{c}$ is a DFT, and, finally, the frequencydomain equalizer (FEQ) block corrects the dispersive effect of the transmission channel.

In DCT-MCM, $\mathbf{T}_{a}^{-1}$ can be any kind of inverse DCT [16], with two different types of redundancy: symmetric extension (SE) or zero-padding (ZP). This work focuses on Type-II even discrete cosine transform (DCT2e) and Type-IV even discrete cosine transform (DCT4e), assuming SE [2, 5]. Unlike DFT-based systems, two symmetrical extensions of $N_{G}$ samples are appended: a prefix or left extension (LE), and a suffix or right extension (RE). Figures 2(b) and 2(c) show examples of the symmetric extensions for DCT-MCM.

In Figure 1, the received sequence in presence of a temporal delay $\delta$ and carrier frequency offset $\epsilon$ can be expressed as

$$
y[n]=\frac{1}{N} \sum_{k=0}^{N-1} H[k] \cdot X[k] \cdot e^{j(2 \pi(k+\varepsilon)(n+\delta) / N)}+z[n],
$$

where $H[k]=\operatorname{DFT}\{h[n]\}=\operatorname{DFT}\left\{h_{c h}[n] * h_{p f}[n]\right\}$ and $z[n]$ is a term related to the noise. An additional constraint of DCT-MCM is that the channel impulse response $h[n]$ must be symmetric, as shown in Figure 2(d). It is important to highlight that some channels satisfy this condition, such as chromatic dispersion in single-mode fibers [4]. If not, the prefilter $h_{p f}[n]$ of Figure 1 is located at the receiver to enforce the symmetry in $h_{c h}[n]$. There are some appropriate techniques to design this prefilter $[2,6]$. For the sake of simplicity, we adopt a model of symmetric discrete-time channel $h[n]$ for DCT-MCM. If this condition is not satisfied, then the training symbol used in [6] can be employed to perform an initial timing symbol synchronization using the algorithms proposed in this paper, and then the channel estimation of $h_{c h}[n]$ can be carried out.

Finally, the channel length of $h_{c h}[n]$ is assumed to be less than the length of the redundancy (SE or CP); i.e., $v+1<N_{G}$. For a more thorough description of DCT-MCM, we refer the reader to $[2,5,16]$.

\section{Proposed Metrics}

An analysis using the correlation between two signals provides a quantitative measure of the similarity between them. In STO estimation, the idea behind [7-9] and other approaches in $[10,11]$ is to use correlation functions to find the similarities that are shared by the data part of the symbol and the redundant samples in the prefix/suffix.

In DCT-MCM, the maximum value or peak is also reached (in the absence of noise) when there exists a set of samples that are pairwise correlated. The time position of this maximum value is useful in finding the symbol timing and the phase of the correlation could yield the frequency estimate. Given that in DCT-MCM the redundancy follows a mirror (anti)symmetry and the channel impulse response is a whole-sample symmetry (WS) sequence, there are two blocks of $N_{G}-v$ samples that are pairwise correlated. For example, let us consider the absence of noise, the fact that $\delta$ is an integer number and $\epsilon=0$, and the time instants represented in Figure 3. At the start of the symbol, specifically at instants $n_{0}-1$ and $n_{0}$, we have

$$
\begin{aligned}
& y\left[n_{0}-1\right]=y\left[n_{0}\right] \\
& =\sum_{k=1}^{v} h[k]\left(x_{e}\left[n_{0}+\delta-k-1\right]+x_{e}\left[n_{0}+\delta+k-1\right]\right) \\
& \quad+h[0] x_{e}\left[n_{0}+\delta-1\right] .
\end{aligned}
$$

In addition, at the end of the symbol, the receiving signal presents identical (for the HS extension) or opposite (for the HA extension) values in two consecutive samples, $n_{0}+N-1$ and $n_{0}+N$. That is, we have

$$
\begin{aligned}
y[ & \left.n_{0}+N-1\right]=\gamma y\left[n_{0}+N\right]=\sum_{k=1}^{\nu} h[k] \\
& \cdot\left(x_{e}\left[n_{0}+N+\delta-k-1\right]\right. \\
& \left.+x_{e}\left[n_{0}+N+\delta+k-1\right]\right)+h[0] x_{e}\left[n_{0}+N+\delta\right. \\
& -1]
\end{aligned}
$$




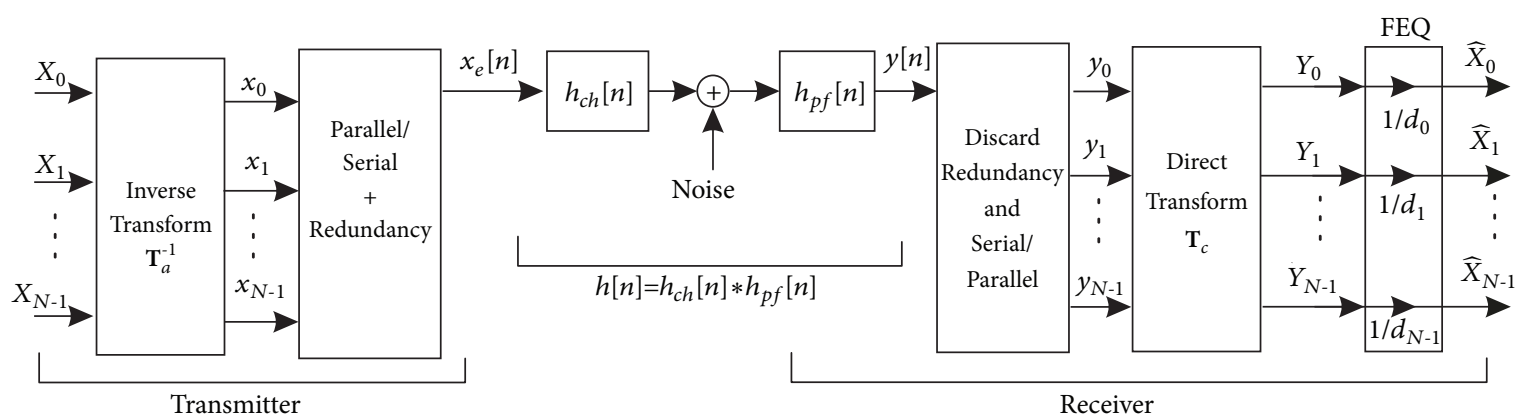

FIGURE 1: Block diagram of a transforms-based multicarrier system over a channel with additive noise.

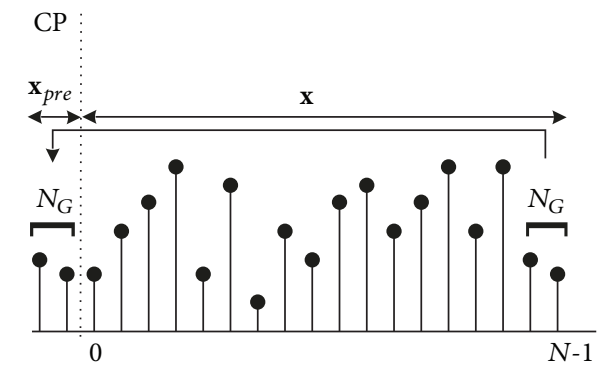

(a)

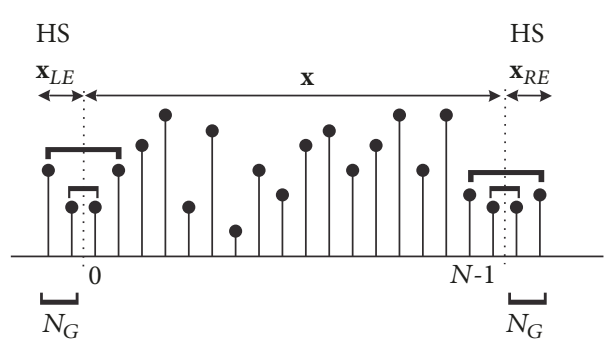

(b)

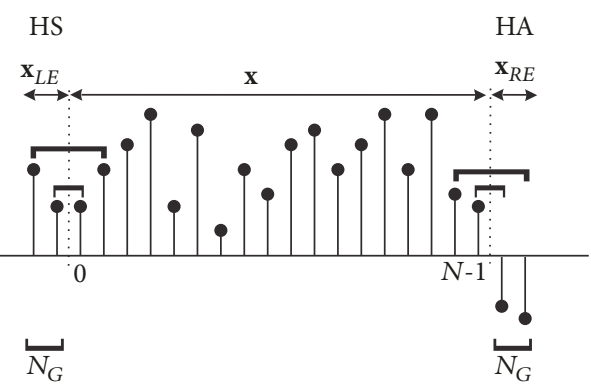

(c)



(d)

FIgure 2: (a) Cyclic prefix for DFT-based systems. Symmetrical extensions for (b) DCT2e-based and (c) DCT4e-based systems. HS and HA stand for half-sample symmetry and half-sample antisymmetry, respectively. (d) Symmetry in the channel impulse response h. WS stands for whole-sample symmetry $(h[n]=h[-n], 1 \leq n \leq \nu)$.

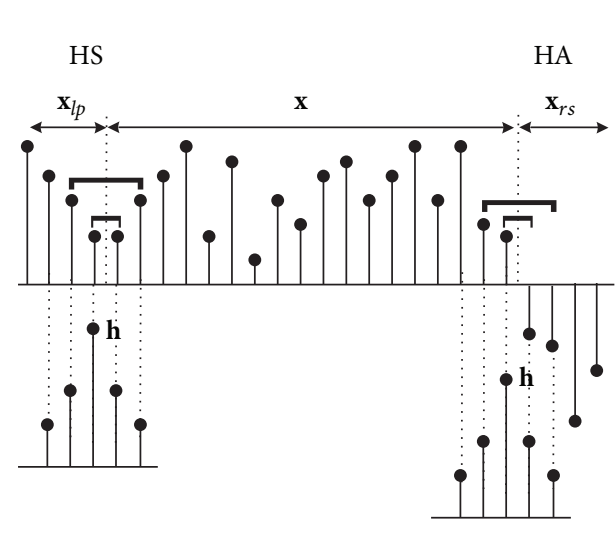

(a)

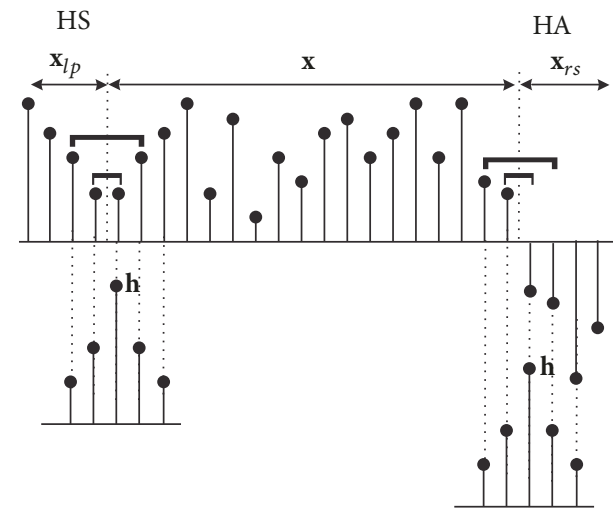

(b)

FIGURE 3: Transmitted symbol and channel impulse response at different instants. (a) $n_{0}-1$ and $n_{0}+N-1$. (b) $n_{0}$ and $n_{0}+N$. ( $N_{G}=4$, $v=2$ ). 
where $\gamma=1$ for the HS extension or $\gamma=-1$ for the HA extension (see Figure 2 and $[2,16]$ ). Notice that there are other sets of $N_{G}-v-1$ identical samples at the start of the received symbol:

$$
y\left[n_{0}-\ell-1\right]=y\left[n_{0}+\ell\right], \quad \delta \leq \ell \leq \delta+N_{G}-v-1,
$$

and identical (for the HS extension) or opposite (for the HA extension) samples at the end of the received symbol:

$$
\begin{aligned}
y\left[n_{0}+N-\ell-1\right]=\gamma y\left[n_{0}+N+\ell\right], & \\
& \delta \leq \ell \leq \delta+N_{G}-v-1 .
\end{aligned}
$$

Considering the above, we propose new blind algorithms to perform tight timing offset and coarse frequency synchronization for DCT2e-MCM and DCT4e-MCM. The first proposed approach estimates the timing offset $\widehat{\delta}_{S E}$ using the maximum of the metric:

$$
M_{L R E_{a}}[\delta]=\left|C_{L E}[\delta]\right|^{2}+\left|C_{R E}[\delta]\right|^{2}-\frac{E_{S E}[\delta]}{4 N_{G}},
$$

where

$$
\begin{aligned}
& C_{L E}[\delta]=\sum_{\ell=\delta}^{\delta+N_{G}-1} y[\ell] y^{*}[-\ell-1], \\
& C_{R E}[\delta]=\sum_{\ell=\delta}^{\delta+N_{G}-1} y[N-1-\ell] \gamma y^{*}[N+\ell] .
\end{aligned}
$$

The normalization term $E_{S E}[\delta]$ considers the energy of the windowed signals:

$$
E_{S E}[\delta]=E_{L E}[\delta]+E_{R E}[\delta],
$$

where

$$
\begin{aligned}
& E_{L E}[\delta]=\left(\sum_{\ell=\delta}^{\delta+N_{G}-1}|y[-\ell-1]|^{2}+\sum_{\ell=\delta}^{\delta+N_{G}-1}|y[\ell]|^{2}\right), \\
& E_{R E}[\delta] \\
& =\left(\sum_{\ell=\delta}^{\delta+N_{G}-1}|y[N-1-\ell]|^{2}+\sum_{\ell=\delta}^{\delta+N_{G}-1}|y[N+\ell]|^{2}\right) .
\end{aligned}
$$

The second proposed method is based on Schmidl and Cox's approach. We adapt the metric of [8] to the scheme

\begin{tabular}{|c|c|}
\hline Parameters & Value \\
\hline System Bandwidth & $5 \mathrm{MHz}$ \\
\hline Sampling Period & $200 \mathrm{~ns}$ \\
\hline Carrier Frequency & $2 \mathrm{GHz}$ \\
\hline $\begin{array}{l}\text { Modulation and } \\
\text { Demodulation }\end{array}$ & 4-QAM \\
\hline $\begin{array}{l}\text { Total Subcarrier Number } \\
(N)\end{array}$ & 512 \\
\hline \multirow{2}{*}{$\begin{array}{l}\text { Length of Redundancy } \\
\text { (Examples } 2 \text { and } 3 \text { ) }\end{array}$} & 32 (OFDM) \\
\hline & $32 \times 2$, Left and Right Prefixes (DCT) \\
\hline \multirow{2}{*}{ Channel Models } & ITU Ped A 4 km/h (PED200) [15] \\
\hline & ITU Veh A 100 km/h (VEH200) [15] \\
\hline Channel Equalization & Zero Forcing \\
\hline Noise Model & iid AWGN \\
\hline Detection & Hard Decision \\
\hline Number of Simulations & 10000 \\
\hline
\end{tabular}
of prefix and suffix used in DCT-MCM, and, as a result, the timing offset can be estimated using the correlation peak given by

$$
M_{L R E_{b}}[\delta]=\frac{\left|C_{L E}[\delta]\right|^{2}}{E_{W_{1 p}, L E}^{2}[\delta]}+\frac{\left|C_{R E}[\delta]\right|^{2}}{E_{W_{1 s}, R E}^{2}[\delta]}
$$

where

$$
\begin{aligned}
& E_{W_{1 p}, L E}[\delta]=\sum_{\ell=\delta}^{\delta+N_{G}-1}|y[\ell]|^{2}, \\
& E_{W_{1 s}, R E}[\delta]=\sum_{\ell=\delta}^{\delta+N_{G}-1}|y[N-1-\ell]|^{2} .
\end{aligned}
$$

TABLE 1: Simulation parameters.

Finally, assuming perfect symbol synchronization at $\widehat{\delta}_{S E}$ and absence of noise, we have that

$$
\begin{aligned}
\left|y\left[\ell+\widehat{\delta}_{S E}\right]\right| & =\left|y^{*}\left[-\ell-1+\widehat{\delta}_{S E}\right]\right|, \\
\left|y\left[\ell+N+\widehat{\delta}_{S E}\right]\right| & =\gamma\left|y^{*}\left[-\ell-1+N+\widehat{\delta}_{S E}\right]\right|,
\end{aligned}
$$

for those pairwise correlated samples. Considering the above condition, a coarse fractional CFO estimator $(|\varepsilon| \leq 0.5)$ for DCT-MCM can be carried out by means of the expression

$$
\widehat{\varepsilon}_{S E}=\frac{N}{2 \pi N_{G}} \angle\left(C_{L E}\left[\widehat{\delta}_{S E}\right]+\gamma C_{R E}\left[\widehat{\delta}_{S E}\right]\right) .
$$

\section{Experimental Study}

In our experiments, we have assumed systems with 512 subcarriers and 4-QAM modulation. Each experiment consists of an average of 10,000 simulation runs with STO independently generated and uniformly distributed over $[-31,0)$. For the first experiment, the length of the redundant samples is modified to study its effects on different parameters. For the rest, we have considered CPs of 32 samples for the DFT-MCM and the same number of redundant samples for each prefix (LE) and suffix (RE) in DCT-MCM. The setup used in our experiments is summarized in Table 1.

Example 1. The influence of the number of redundant samples $\left(N_{G}\right)$ is studied. The performance of the proposed estimators (6)-(10), for both DCT2e and DCT4e, is assessed and compared with that derived in [10] based on [7], for SNR values of 10 and $16 \mathrm{~dB}$ and over discrete memoryless additive white Gaussian noise (AWGN) channels, with no intersymbol interference. The SNR is defined by the ratio of the power of 


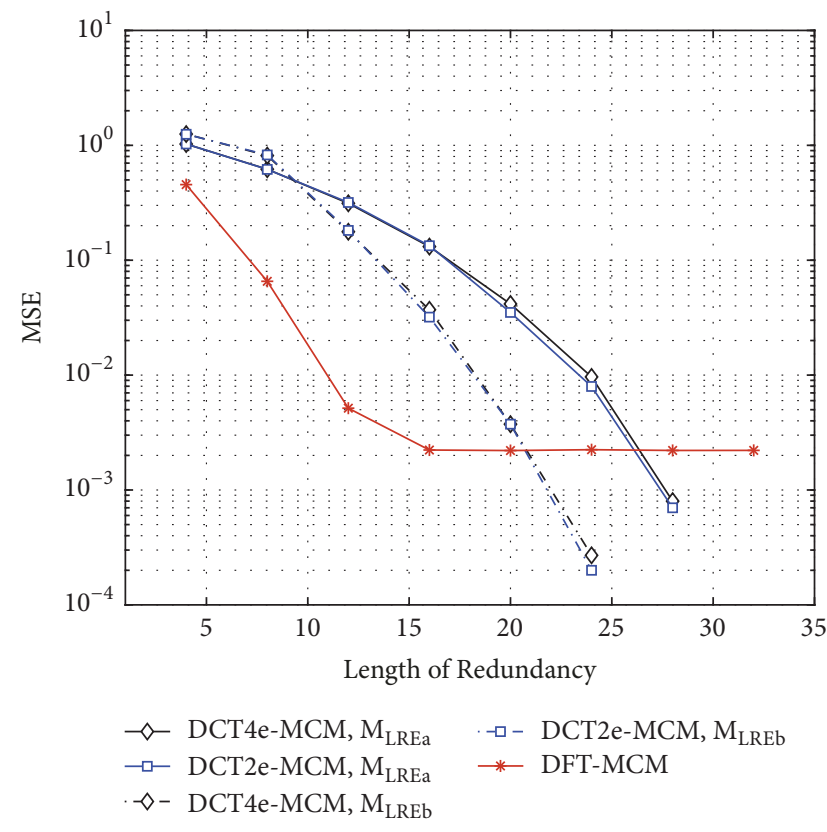

(a)

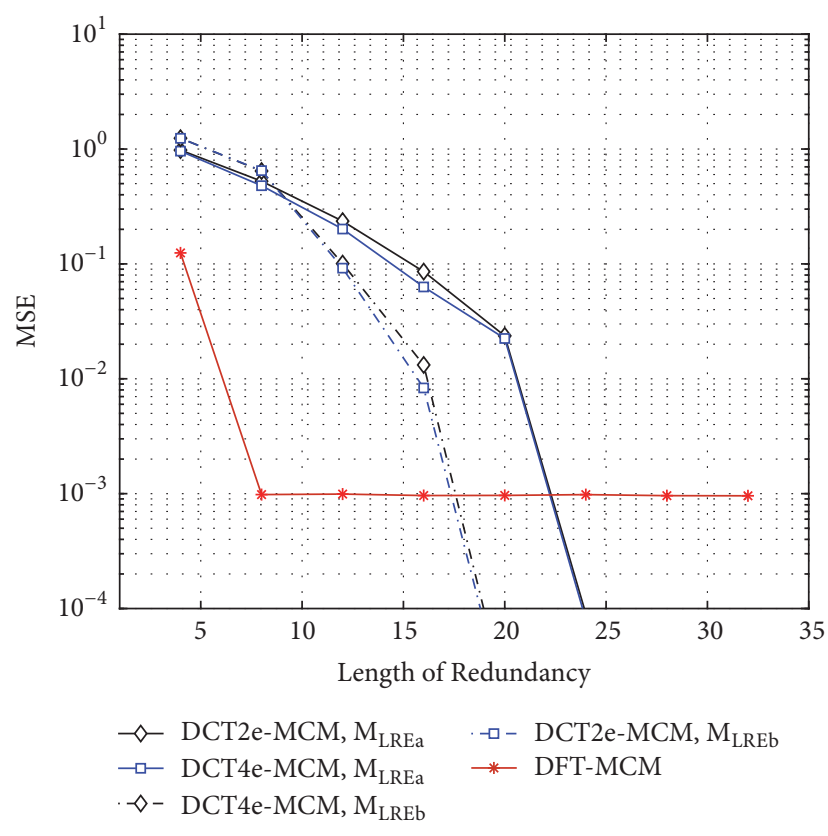

(b)

FIGURE 4: Example 1. MSE of STO as an estimator for the AWGN channel and different lengths of the redundancy: (a) SNR = $10 \mathrm{~dB}$, (b) SNR $=16 \mathrm{~dB}$.

the received signal and the variance of the AWGN. Figure 4 shows the resulting mean-square error (MSE) of the STO estimates evaluating the trial and the estimate values of the timing offset. As was documented in [7], it can be seen in Figure 4 that the performance of the DFT-MCM estimator is asymptotically independent of the number of redundant samples, provided that the amount of data for the $\mathrm{CP}$ is longer than a certain threshold value, which decreases with the SNR (see Figure 4 and $[7,10]$ ). As the length increases beyond the thresholds, the DFT-MCM time estimator does not improve. On the contrary, the behaviour of the proposed schemes for DCT-MCM is no longer flat. MSE decays monotonically and the improvement is achieved by increasing the SNR and/or the number of redundant samples. Note that MSEs for the DFT-MCM saturate at $2.2 \cdot 10^{-3}$ and $9.9 \cdot 10^{-4}$, and that the performance of the proposed schemes is much better, with MSE $<10^{-3}$, when SNR $=16$ and the number of redundant data is greater than 22 (see (6)) or 17 (see (10)).

We also use our analysis to show how BER changes with the length of the redundant samples. Figure 5 depicts the uncoded BER curves, and, given that the results have been practically indistinguishable for DCT2e and DCT4e, only one curve to represent DCT-MCM is included. Notice that the BER curves become practically saturated for DFT-MCM, and increasing the number of redundant samples does not improve BER. An immediate conclusion that can be drawn from these plots is that the proposed metrics exhibit good performance in the estimation of the STO when the length of the redundant samples is at least one-half of the maximum considered offset. For DCT-MCM, the performance of the proposed approaches for $\mathrm{SNR}=10 \mathrm{~dB}$ does not improve as

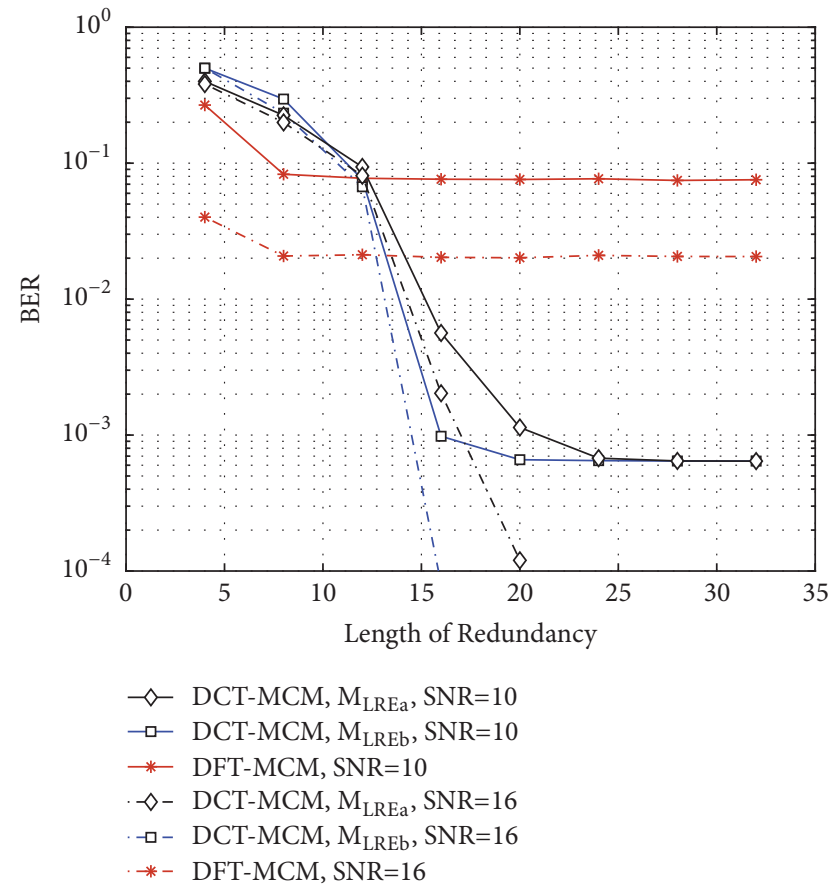

FIgURE 5: Example 1. BER under conditions of STO for different length for the redundancy and SNR values considering AWGN channels.

the length of the redundant sample increases from 20 to 32 , whereas it is possible to obtain BER values lower than $10^{-4}$ for $\mathrm{SNR}=16 \mathrm{~dB}$. 

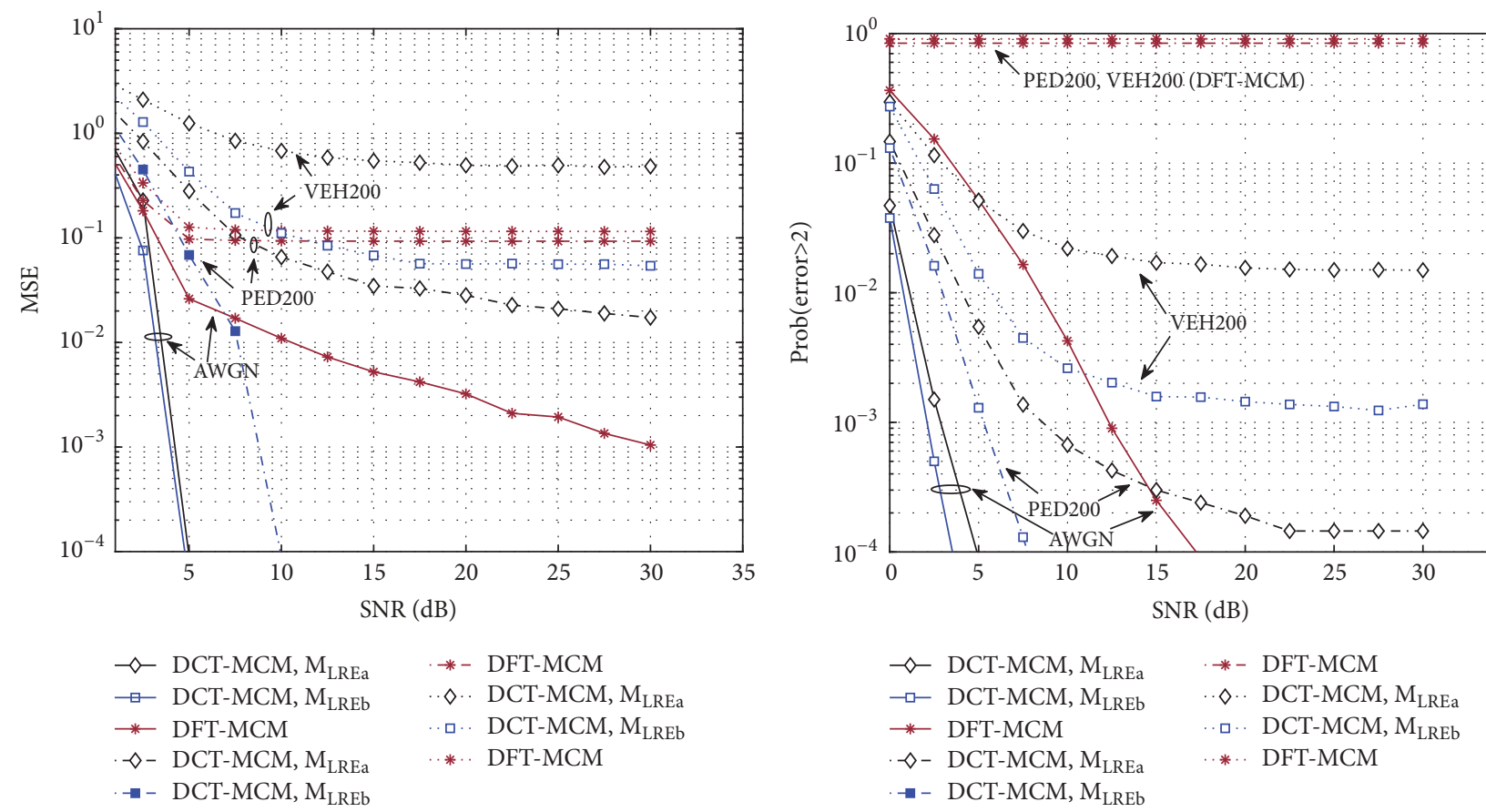

(a)

(b)

FIGURE 6: Example 2. Effects of STO for different transceivers and channels. (a) Mean-square error. (b) Probability of failure.

Example 2. In our second set of experiments, all of the results are obtained over three different channels: AWGN and two sets of 100 wireless fading channels each, according to the ITU Pedestrian A and Vehicular A channels [15]. The multipath channels were generated with Matlab's stdchan using the channel models itur3GPAx and itur3GVAx with a carrier frequency $f_{c}=2 \mathrm{GHz}$ and two different sets of parameters: (a) $4 \mathrm{~km}$ per hour as pedestrian velocity, $T_{s}=200 \mathrm{~ns}$ and length $L=11$; and (b) $100 \mathrm{~km}$ per hour as mobile speed (the moving speed has been chosen considering that the average vehicle speed on European highways lies in the range of 90$100 \mathrm{~km}$ per hour under free-flow conditions), $T_{s}=200 \mathrm{~ns}$ and length $L=21$. These channels are referred to as PED200 and VEH200, respectively.

Figure 6(a) shows the resulting mean-square error (MSE) of the STO estimates. As can be seen, the performance of $M_{L R E_{b}}$ is much better than the others, with the exception of the VEH200 channel, for which [7] provides the best values of MSE.

The estimated temporal offsets have also been obtained. Based on the fact that an accuracy of less than three samples at low SNR makes the approach robust in fading channels $[7,10]$, we have obtained the probability that the estimated error in the time offset is greater than two samples. The numerical results for the three different transceivers over the channels are displayed in Figure 6(b). These curves show that DCT-based methods significantly improve the performance compared to the DFT-MCM over the entire range of SNR values considered in our experiments.

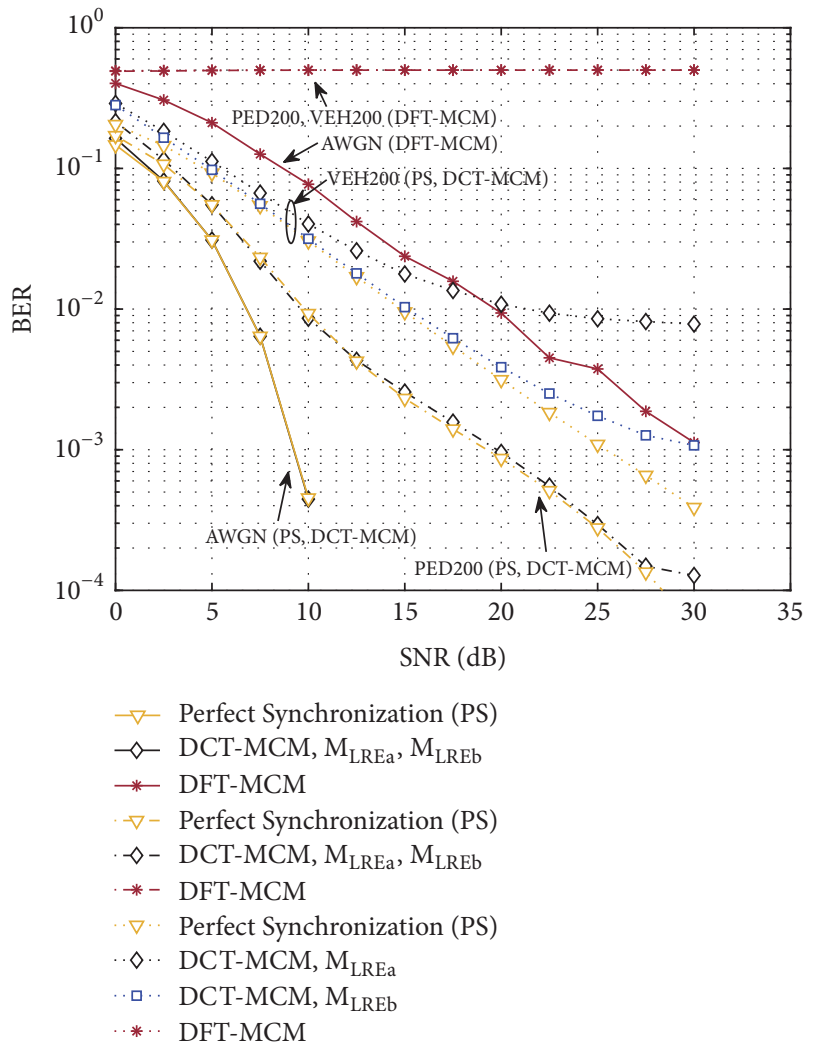

FIGURE 7: Example 2. BER under symbol timing offset for different transceivers and channels. 


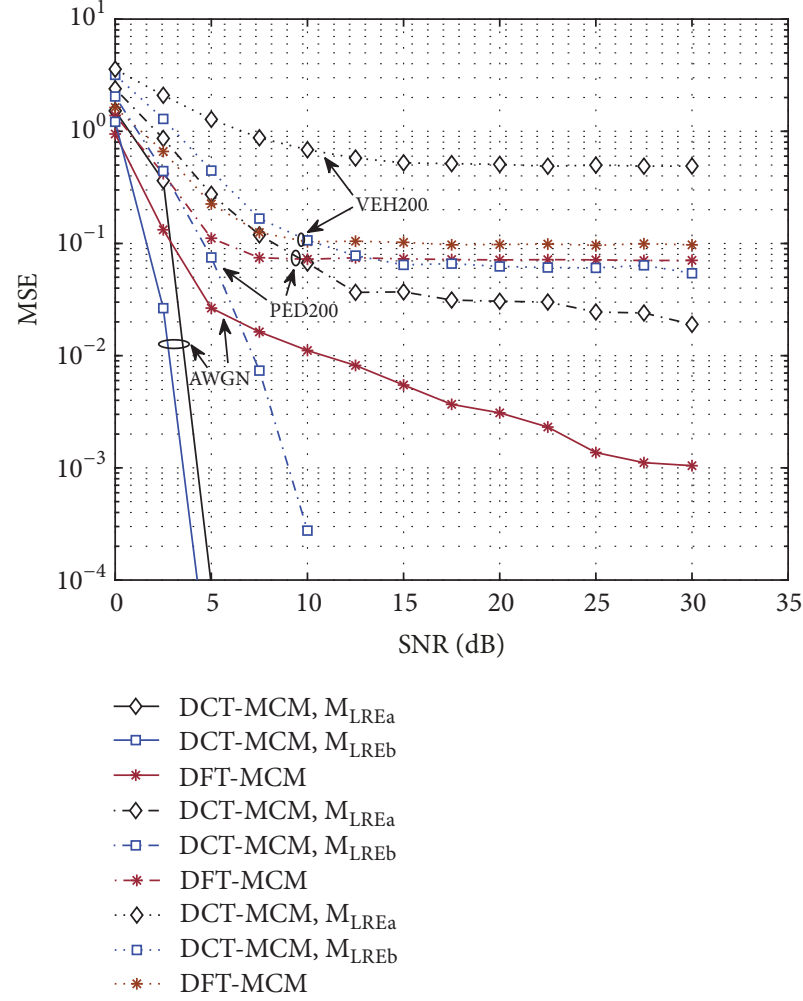

(a)

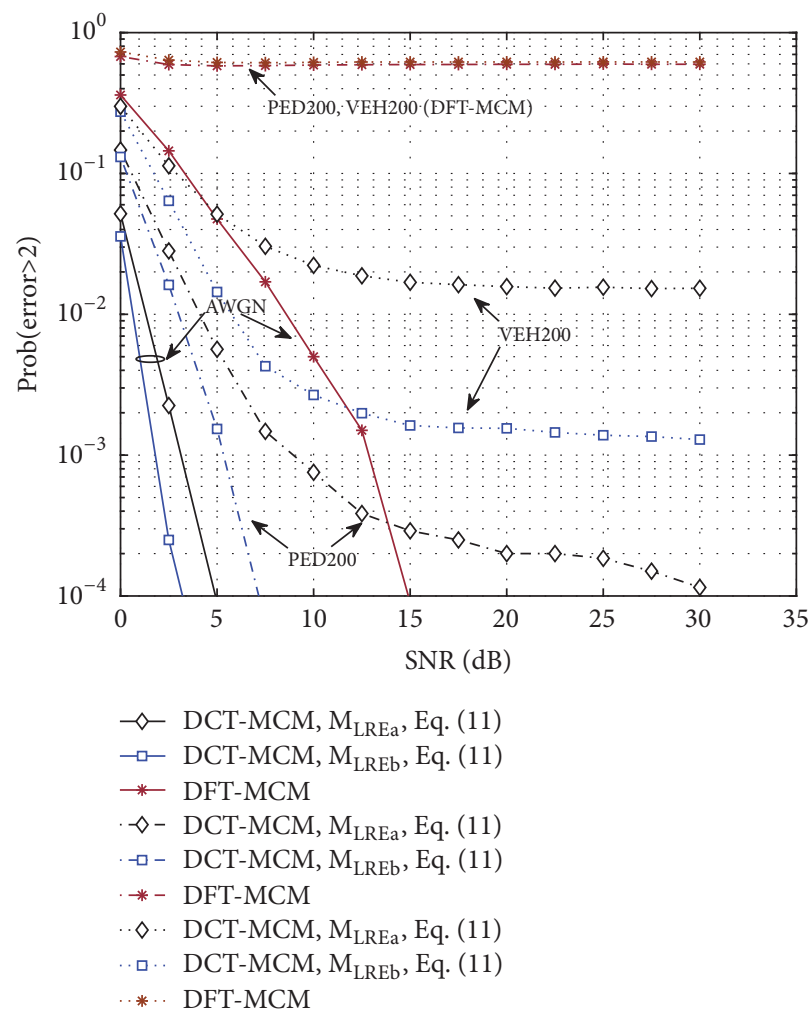

(b)

FIGURE 8: Example 3. Effects of STO and CFO for different transceivers. (a) Mean-square error of time offset estimate. (b) Probability of failure for time offset estimate.

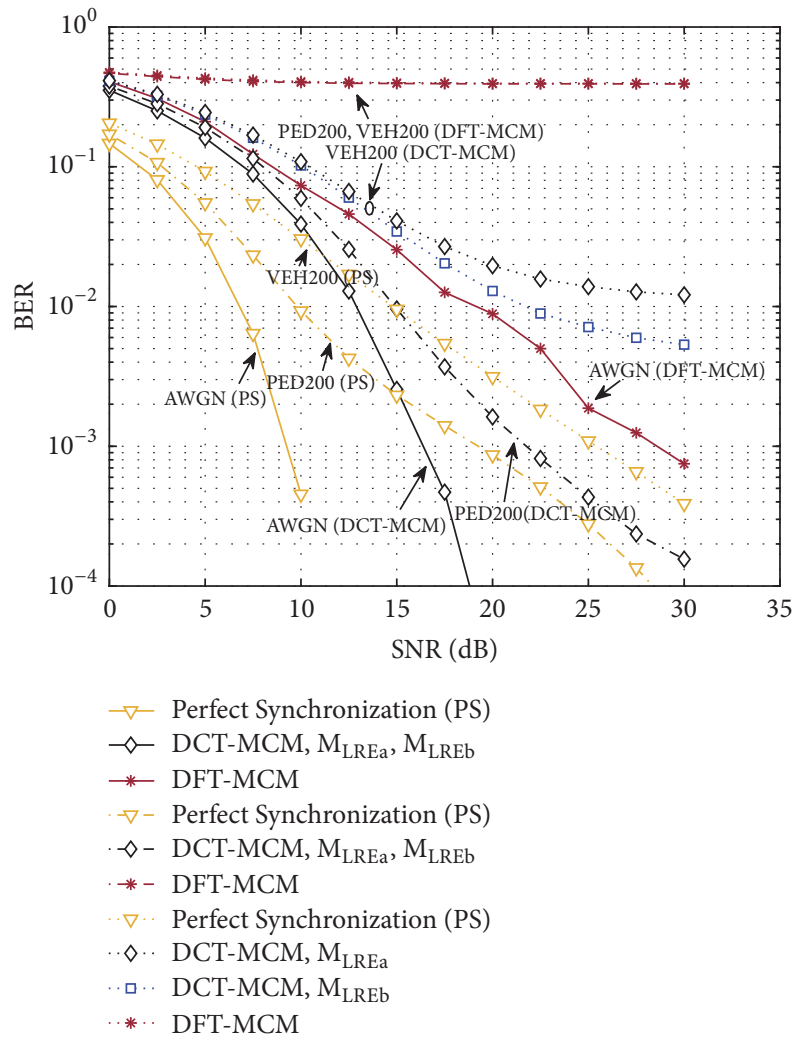

FIgurE 9: Example 3. BER under the effects of STO and CFO for different channels and transceivers.
Next, we compare the BER performance for the three different channels. Figure 7 depicts the obtained results versus the received SNR. In this figure, perfect synchronization (PS) represents the case where STO is perfectly known at the receiver. Interestingly, for a particular channel, the BER performance of the proposed metrics $M_{L R E_{a}}$ and $M_{L R E_{b}}$ is practically indistinguishable, and for this reason we only represent one curve. In addition, the proposed methods have the best performance. They are close to the perfect synchronization for the AWGN and PED200 channels, and a performance degradation occurs for the VEH200 channel. On the other hand, the DFT-MCM system is severely affected by a timing offset, especially for the PED200 and VEH200 channels.

Example 3. Lastly, we consider a more complex scenario, where a normalized CFO is added following a uniform distribution in the interval $[-0.04,0)$, which is estimated by the approach in [7] for DFT-MCM, whereas (13) is employed for DCT-MCM. Figures 8(a) and 8(b) characterize the MSE and $\operatorname{Pr}(|\delta-\widehat{\delta}|)>2$ of the STO estimates, respectively, whereas Figure 9 plots the resulting BER. Comparing the performance of the proposed algorithms with DFT-MCM, it is proved that the new approaches work properly in AWGN and PED200 channels, significantly improving the performance and reducing the BER. The proposed schemes also perform well for the VEH200 channel, although in this case the CFO has an effect on our schemes. On the other 
hand, the BER is practically flat for DFT-MCM in the more challenging realistic scenarios of PED200 and VEH200.

\section{Conclusions}

DCT-MCM offers benefits such as excellent spectral compaction and energy concentration and allows a better usage of the spectrum. In this work, new techniques of symbol timing estimation for DCT-MCM systems are proposed. The new metrics are based on the fact that the correlation between two signals provides a quantitative measure of their similarity, and they take into account the fact that the redundant samples in DCT-MCM are inserted as left (prefix) and right (suffix) extensions satisfying (anti)symmetry properties. A wide set of simulations have been carried out to test the effectiveness of the proposed approaches. It can be established from the results of our experiments that the proposed techniques perform well, especially in Gaussian and low dispersive channels. It is further shown that considering the BER and the probability of the estimate error in the time offset, a performance gain over the conventional DFT-based technique is obtained when the length of the redundancy increases.

\section{Data Availability}

The data used to support the findings of this study are not needed actually. The performance study was based on the data randomly generated by the simulation code.

\section{Conflicts of Interest}

The authors declare that there are no conflicts of interest regarding the publication of this article.

\section{Acknowledgments}

This work was partially supported by the Spanish Ministry of Economy and Competitiveness through Project TEC201564835-C3-1-R.

\section{References}

[1] J. Cioffi, "Multichannel modulation," in Digital Communications, https://web.stanford.edu/group/cioffi/doc/book/chap4 .pdf.

[2] N. Al-Dhahir, H. Minn, and S. Satish, "Optimum DCTbased multicarrier transceivers for frequency-selective channels," IEEE Transactions on Communications, vol. 54, no. 5, pp. 911-921, 2006.

[3] P. Tan and N. C. Beaulieu, "A comparison of DCT-based OFDM and DFT-based OFDM in frequency offset and fading channels," IEEE Transactions on Communications, vol. 54, no. 11, pp. 2113-2125, 2006.

[4] X. Ouyang and J. Zhao, "Single-tap equalization for fast OFDM signals under generic linear channels," IEEE Communications Letters, vol. 18, no. 8, pp. 1319-1322, 2014.

[5] F. Cruz-Roldán, M. E. Domínguez-Jiménez, G. Sansigre-Vidal, J. Pineiro-Ave, and M. Blanco-Velasco, "Single-carrier and multicarrier transceivers based on discrete cosine transform type-IV," IEEE Transactions on Wireless Communications, vol. 12, no. 12, pp. 6454-6463, 2013.

[6] F. Cruz-Roldán, M. E. Domínguez-Jiménez, G. Sansigre-Vidal, D. Luengo, and M. Moonen, "DCT-based channel estimation for single- and multicarrier communications," Signal Processing, vol. 128, pp. 332-339, 2016.

[7] J.-J. Van De Beek, M. Sandell, and P. O. Börjesson, "ML estimation of time and frequency offset in OFDM systems," IEEE Transactions on Signal Processing, vol. 45, no. 7, pp. 18001805, 1997.

[8] T. M. Schmidl and D. C. Cox, "Robust frequency and timing synchronization for OFDM," IEEE Transactions on Communications, vol. 45, no. 12, pp. 1613-1621, 1997.

[9] T. Kung and K. K. Parhi, "Optimized joint timing synchronization and channel estimation for OFDM systems," IEEE Wireless Communications Letters, vol. 1, no. 3, pp. 149-152, 2012.

[10] Y. S. Cho, J. Kim, W. Y. Yang, and C. G. Kang, MIMO-OFDM Wireless Communications with Matlab, IEEE Press, John Wiley \& Sons, 2010.

[11] A. A. Nasir, S. Durrani, H. Mehrpouyan, S. D. Blostein, and R. A. Kennedy, "Timing and carrier synchronization in wireless communication systems: a survey and classification of research in the last 5 years," EURASIP Journal on Wireless Communications and Networking, vol. 2016, no. 1, article 180, 2016.

[12] T. H. Pham, V. A. Prasad, and A. S. Madhukumar, "A HardwareEfficient Synchronization in L-DACS1 for Aeronautical Communications," IEEE Transactions on Very Large Scale Integration (VLSI) Systems, vol. 26, no. 5, pp. 924-932, 2018.

[13] F. Gao, T. Cui, A. Nallanathan, and C. Tellambura, "Maximum likelihood based estimation of frequency and phase offset in DCT OFDM systems under non-circular transmissions: algorithms, analysis and comparisons," IEEE Transactions on Communications, vol. 56, no. 9, pp. 1425-1429, 2008.

[14] J. Zhao, S. K. Ibrahim, D. Rafique, P. Gunning, and A. D. Ellis, "Symbol synchronization exploiting the symmetric property in optical fast OFDM," IEEE Photonics Technology Letters, vol. 23, no. 9, pp. 594-596, 2011.

[15] 3GPP, “Technical specification group radio radio access network. user equipment (UE) radio transmission and reception (FDD) (release 7)," 3GPP TS 3GPP TS 25.101, 3rd Generation Partnership Project, 2007.

[16] F. Cruz-Roldán, M. E. Domínguez-Jiménez, G. Sansigre-Vidal, P. Amo-López, M. Blanco-Velasco, and A. Bravo-Santos, "On the use of discrete cosine transforms for multicarrier communications," IEEE Transactions on Signal Processing, vol. 60, no. 11, pp. 6085-6090, 2012. 


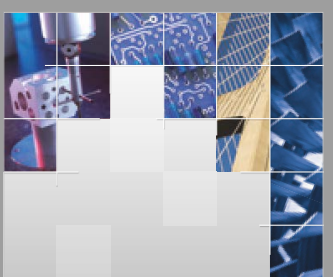

\section{Enfincering}
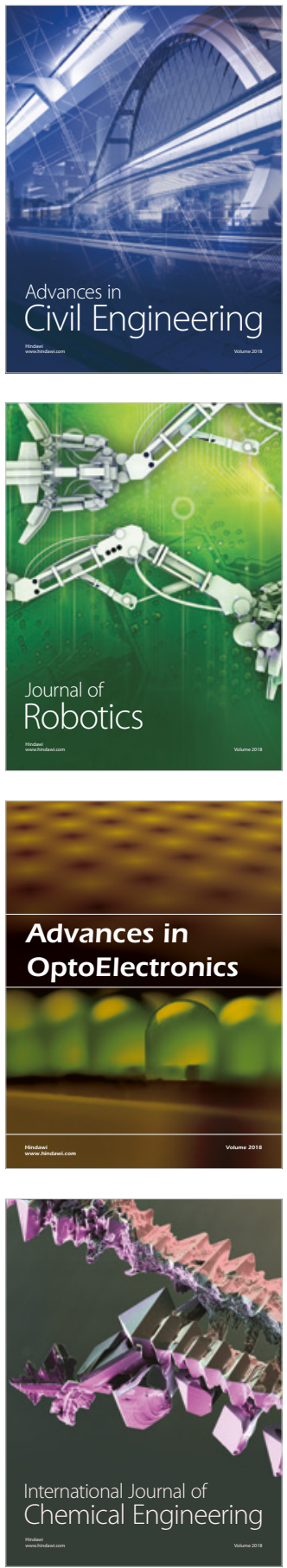

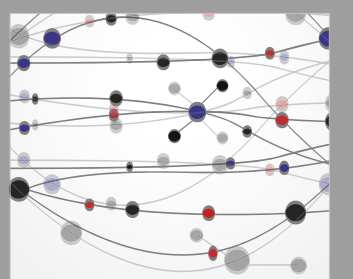

\section{Rotating \\ Machinery}

The Scientific World Journal

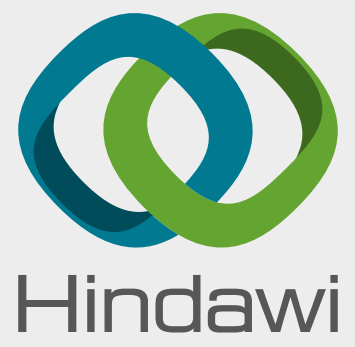

Submit your manuscripts at

www.hindawi.com
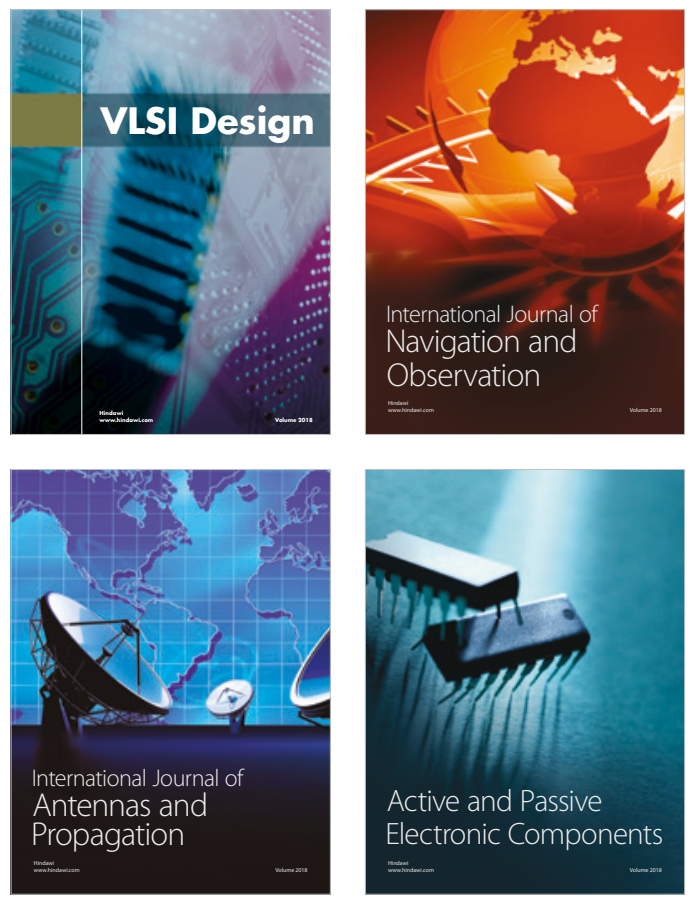
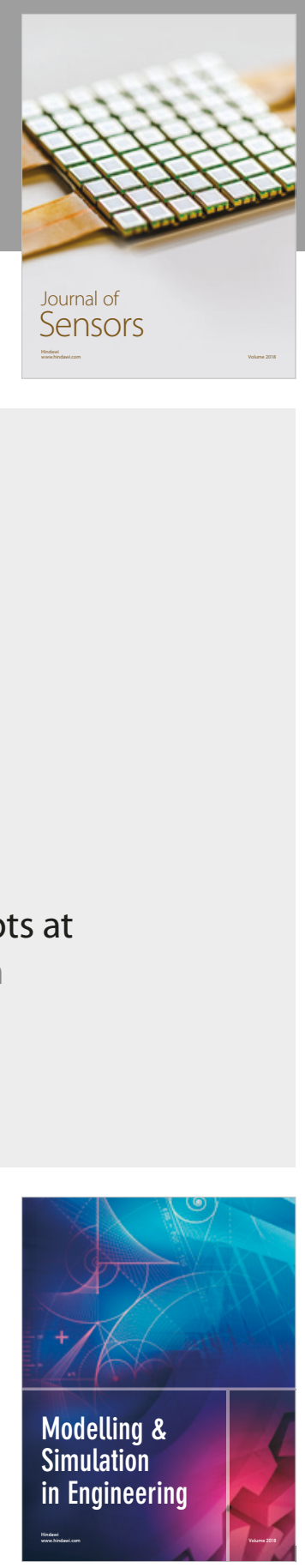

\section{Advances \\ Multimedia}
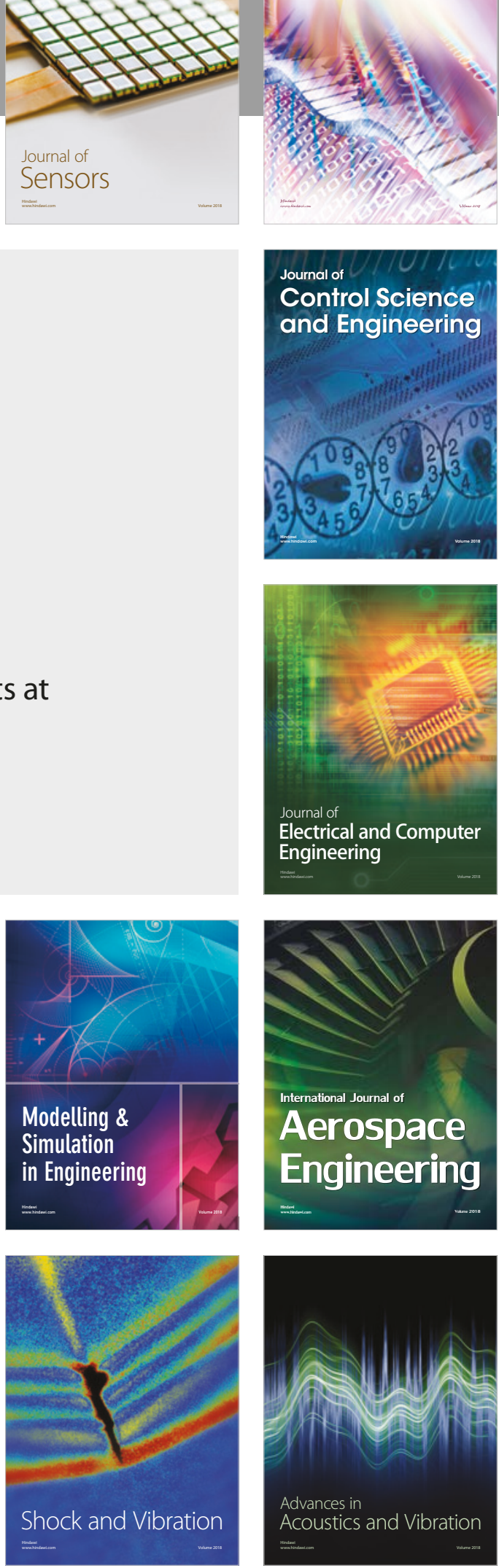